\title{
Assessment of enclosing structure moisture regime using moisture potential theory
}

\author{
Vladimir Gagarin ${ }^{1,2}$, Vadim Akhmetov ${ }^{1}$, and Kirill Zubarev ${ }^{1, *}$ \\ ${ }^{1}$ Moscow State University of Civil Engineering, 26, Yaroslavskoe shosse, 129337, Moscow, Russia \\ ${ }^{2}$ Research Institute of Building Physics of Russian Academy of Architecture and Construction \\ Science, 21, Locomotive travel, 127238, Moscow, Russia
}

\begin{abstract}
The paper describes principal development directions of mathematical models of enclosing structure moisture regime. Benefits of mathematical models based on moisture potential theory are demonstrated. Moisture regime calculation by means of moisture potential, taking liquid and vapor moisture transfer into consideration, and using discretecontinual approach is proposed. New formulas for single-layer and multilayer enclosing structures allowing for numerical analytic determination of moisture potential value in any enclosing structure section, at any moment of time, under continuous control of temperature distribution, have been derived. Moisture distribution has been studied for a number of enclosing structures using different methods of moisture potential theory: unsteadystate method, quasi-stationary method, the proposed discrete-continual method. Moisture regime has been determined for single-layer enclosing structure with ceramic brick basement and lime brick cladding in Moscow. It is shown that the greatest moisture value is achieved in enclosing structure calculation by means of steady-state method. Unsteady-state method gives more accurate moisture distribution. The proposed discretecontinual method gives quantitative and qualitative result of moisture distribution similar to results obtained by unsteady-state method. The benefit of discrete-continual method is a distribution obtained analytically, which allows to use solution results without numerical method application.
\end{abstract}

\section{Introduction}

\subsection{Moisture regime and its role in construction}

Assessment of enclosing structure moisture regime is one of the key directions in construction. Errors in overwetting protection design can reduce enclosing structure durability and result in premature failure of the building [1-5]. Moisture in enclosing structure thickness influences both on power saving properties of building thermal protective shell [6-11], and on human health [12].

Experimental test methods for moisture diffusion coefficients are being actively developed at present [13-17], new laboratory equipment is developed and implemented in

\footnotetext{
* Corresponding author: zubarevkirill93@mail.ru
} 
researches [18-20], on-site measurements of enclosing structures moisture state are carried out $[21,22]$.

Design of methods for enclosing structure moisture regime calculation is one of the important development directions. There are two principal approaches to this issue: methods based on separate calculation of moisture transfer schemes [23-26], and methods based on moisture potential theory [27].

\subsection{Moisture transfer in sorption wetting zone}

First methods of moisture regime calculation appeared in 1920s. Well-known "consequent wetting method" is an example. It is expected that moisture transfer is carried out under water vapor partial pressure gradient. Moisture flow in the enclosing structure is a flow of vapor moisture.

Moisture transfer differential equation is written as:

$$
\gamma_{0} \cdot \frac{\xi_{0}(w)}{E_{t}(t)} \cdot \frac{\partial e(w, t)}{\partial \tau}=\frac{\partial}{\partial x}\left(\mu \cdot \frac{\partial e(w, t)}{\partial x}\right) .
$$

where e - water vapor partial pressure, $\mathrm{Pa} ; E_{t}$ - saturated water vapor pressure, $\mathrm{Pa} ; \mu-$ vapor permeability coefficient, $\mathrm{kg} /(\mathrm{m} \cdot \mathrm{s} \cdot \mathrm{Pa}) ; \gamma_{0}$ - dry material density, $\mathrm{kg} / \mathrm{m}^{3} ; \xi_{0}$ - relative vapor capacity, $\mathrm{kg} / \mathrm{kg}$; $\mathrm{w}$ - material humidity, $\%$ by weight; $\tau$ - time, $\mathrm{s} ; \mathrm{x}$ - coordinate, $\mathrm{m}$; $\mathrm{t}$ - temperature, ${ }^{\mathrm{C}} \mathrm{C}$.

Third-order boundary conditions of moisture exchange are set at the enclosing structure boundaries:

$$
\begin{array}{r}
-\left.\mu_{1} \frac{\partial e}{\partial x}\right|_{i=1}=\beta_{\text {ext }}\left(e_{\text {ext }}-e_{1}\right) . \\
\left.\mu_{2} \frac{\partial e}{\partial x}\right|_{i=N}=\beta_{i n}\left(e_{i n}-e_{N}\right) .
\end{array}
$$

where $e_{e x t}-$ partial pressure of inside air water vapor, $\mathrm{Pa} ; e_{1}-$ partial pressure of water vapor which contacts with outside air of the enclosing structure section, $\mathrm{Pa} ; \beta_{\text {ext }}-$ moisture exchange coefficient of outside air and enclosing structure surface, $\mathrm{kg} /\left(\mathrm{m}^{2} \cdot \mathrm{s} \cdot \mathrm{Pa}\right)$, $\mu_{1}$ - vapor permeability coefficient of enclosing structure layer which is the nearest to the building outside surface, $\mathrm{kg} /(\mathrm{m} \cdot \mathrm{s} \cdot \mathrm{Pa}), e_{i n}$ - inside air partial pressure, $\mathrm{Pa} ; e_{N}$ - partial pressure of material water vapor which contacts with inside air of enclosing structure surface, $\mathrm{Pa} ; \beta_{i n}-$ moisture exchange coefficient of inside air and enclosing structure surface, $\mathrm{kg} /\left(\mathrm{m}^{2} \cdot \mathrm{s} \cdot \mathrm{Pa}\right), \mu_{2}$ - vapor permeability coefficient of enclosing structure layer which is the nearest to the enclosing structure inner surface, $\mathrm{kg} /(\mathrm{m} \cdot \mathrm{s} \cdot \mathrm{Pa})$.

Condition of water vapor partial pressure flow continuity is set at boundaries between enclosing structure layers: 


$$
-\left.\mu_{1} \frac{\partial e}{\partial x}\right|_{i=v-0}=-\left.\mu_{2} \frac{\partial e}{\partial x}\right|_{i=v+0}
$$

where $\mathrm{v}$ - section of multi-layer enclosing structure, in which there is a material joint.

A constraint resulted from process physics is imposed on water vapor partial pressure:

$$
e \leq E_{t} \text {. }
$$

Saturated water vapor pressure depends on temperature and is described by ClausiusClapeyron equation:

$$
E_{t}=1.84 \cdot 10^{11} \cdot \exp (-5330 /(273+t))
$$

This method is hard to use as temperature distribution varies continuously during enclosing structure operation, and thus, water vapor condensation pressure changes every moment of time, which complicates calculation. The following method is used to overcome such complexity: steady-state thermal conductivity is studied within a timeframe (for example, a month), and then researches proceed to the next timeframe:

$$
\frac{\partial}{\partial x}\left(\lambda \cdot \frac{\partial t}{\partial x}\right)=0 .
$$

where $\lambda$ - thermal conductivity coefficient, $\mathrm{W} /\left(\mathrm{m}^{\circ} \mathrm{C}\right)$.

Moisture continuity provision is set between timeframes:

$$
\left.w\right|_{\tau=\sigma-0}=\left.w\right|_{\tau=\sigma+0} .
$$

where $\sigma-$ moment of time including gap between timeframes, $\mathrm{s}$.

\subsection{Combined moisture transfer in sorption and over-sorption wetting zones}

The described approach allows moisture transfer process calculation in sorption wetting zone, however if water vapor condenses in any part of the structure, liquid moisture capillary flow is added to water vapor transfer. Total moisture flow in the enclosing structure is written as:

$$
g_{\text {tot }}=g_{v}+g_{L}
$$

where $g_{\text {tot }}$ - total moisture flow, $\mathrm{kg} /\left(\mathrm{m}^{2} \cdot \mathrm{s}\right), g_{v}$ - vapor moisture flow, $\mathrm{kg} /\left(\mathrm{m}^{2} \cdot \mathrm{s}\right), g_{L}-$ liquid moisture flow, $\mathrm{kg} /\left(\mathrm{m}^{2} \cdot \mathrm{s}\right)$.

The following equation is used to study moisture transfer in over-sorption zone:

$$
\gamma_{0} \frac{\partial w}{\partial \tau}=\frac{\partial}{\partial x}\left(\beta(w) \frac{\partial w}{\partial x}\right)+\frac{\partial}{\partial x}\left(\mu \frac{\partial E_{t}}{\partial x}\right) .
$$

where $\beta$ - moisture conductivity coefficient, $\mathrm{kg} /(\mathrm{m} \cdot \mathrm{s} \cdot \mathrm{kg} / \mathrm{kg})$.

At the joint of two contacting materials located in over-sorption zone, liquid moisture distributes in proportion to daily average speed of capillary suction.

The equation (10) is valid for an enclosing structure part which is located in oversorption zone. The equation (1) keeps to be valid for sorption wetting zone. Thus moisture transfer schemes are calculated separately. Calculation results obtained by above- 
mentioned method have been confirmed by experiments using on-site moisture state measurements of operating buildings.

There are similar methods taking into consideration a large number of moisture transfer schemes.

Disadvantage of this approach is the need to solve a system of complicated differential equations. The more factors are taken into consideration, the more equations are needed to be solved.

\subsection{Moisture potential application for uniform metering of liquid and vapor moisture flow}

Moisture potential theory has been developed to overcome difficulties described above. Within a frame of above-mentioned model of combined liquid and vapor moisture transfer, V. G. Gagarin's moisture potential [28] has been introduced. This moisture potential takes into account water vapor flow scheme in sorption wetting zone and moisture capillary flow scheme in over-sorption wetting zone:

$$
F(w, t)=E_{t}(t) \cdot \varphi(w)+\frac{1}{\mu} \int_{0}^{w} \beta(\zeta) d \zeta .
$$

where $\mathrm{F}$ - moisture potential, $\mathrm{Pa}, \varphi$ - relative air humidity in material pores.

Moisture potential $\mathrm{F}$ is a function of two variables: humidity and temperature. From a physics perspective the moisture potential reflects total value of water vapor pressure and equivalent liquid moisture pressure.

In graphic form the moisture potential can be presented by three-dimensional scale called "moisture potential scale". For example, moisture potential scale for silica brick has the following form (Fig. 1).

Moisture transfer differential equation made with respect to moisture potential $\mathrm{F}$ is written as:

$$
\gamma_{0} \cdot\left(\frac{1}{\mu} \beta(w)+\frac{\partial \varphi(w)}{\partial w} E_{t}(t)\right)^{-1} \frac{\partial F(w, t)}{\partial \tau}=\frac{\partial}{\partial x}\left(\mu \frac{\partial F(w, t)}{\partial x}\right) .
$$

Assuming that outside and inside air moisture potential is equal to relative elasticity of outside and inside air correspondingly, it can be written as:

$$
F_{\text {in }}=e_{\text {in }} \text {. }
$$

where $F_{i n}$ - inside air moisture potential, $\mathrm{Pa}$.

$$
F_{e x t}=e_{e x t} .
$$

where $F_{\text {ext }}$ - outside air moisture potential, $\mathrm{Pa}$. 


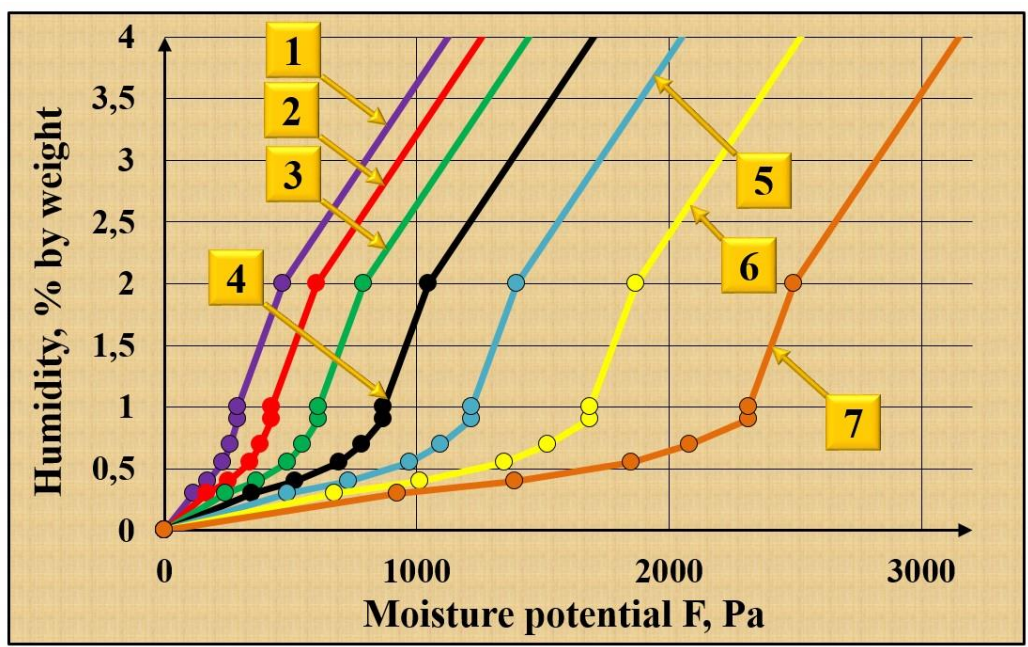

Fig. 1. Moisture potential scale of lime brick $\left(1--10^{\circ} \mathrm{C}\right.$ temperature; $2--^{\circ} \mathrm{C}$ temperature; $3-0^{\circ} \mathrm{C}$ temperature; $4-5^{\circ} \mathrm{C}$ temperature; $5-10^{\circ} \mathrm{C}$ temperature; $6-15^{\circ} \mathrm{C}$ temperature; $7-20^{\circ} \mathrm{C}$ temperature).

Third-order boundary conditions on enclosing structure surface can be written as:

$$
\begin{gathered}
-\left.\mu_{1} \frac{\partial F}{\partial x}\right|_{i=1}=\beta_{e x t}\left(F_{e x t}-F_{1}\right) . \\
\left.\mu_{2} \frac{\partial F}{\partial x}\right|_{i=N}=\beta_{i n}\left(F_{i n}-F_{N}\right) .
\end{gathered}
$$

where $F_{1}$ - moisture potential of enclosing structure section, which contacts with outside air, $\mathrm{Pa}, F_{N}$ - material moisture potential next to enclosing structure surface, which contacts with inside air, $\mathrm{Pa}$.

Provision for moisture potential flow continuity is set at boundaries between enclosing structure layers:

$$
-\left.\mu_{1} \frac{\partial F}{\partial x}\right|_{i=v-0}=-\left.\mu_{2} \frac{\partial F}{\partial x}\right|_{i=v+0} .
$$

Temperature distribution is a quasi-stationary problem according to the equation (7). Moisture continuity is set by equation (8) between timeframes.

The described approach estimates unsteady-state moisture regime of the enclosing structure, and at the same time does not require to solve a system of moisture transfer differential equations as opposed to other methods based on separate consideration of moisture transfer schemes. However, equation (12) requires numerical methods that make calculation more complex.

Steady-state moisture potential distribution is used for engineering calculation:

$$
\frac{\partial}{\partial x}\left(\mu \frac{\partial F(w, t)}{\partial x}\right)=0 .
$$


The equation (18) supports analytical assessment of the moisture regime, but has considerably less possibilities as compared to unsteady-state problem solution.

\section{The problem}

The aim of the research is to develop calculation method of unsteady-state moisture regime for single-layer and multi-layer enclosing structure using discrete-continual approach.

\section{Materials and methods}

\subsection{Rearrangement of moisture transfer equation}

Let us write the equation (12) as follows:

$$
\gamma_{0} \cdot\left(\frac{\partial\left(\varphi(w)+\frac{1}{\mu \cdot E_{t}(t)} \int_{0}^{w} \beta(\zeta) d \zeta\right)}{\partial w} E_{t}(t)\right)^{-1} \frac{\partial F(w, t)}{\partial \tau}=\frac{\partial}{\partial x}\left(\mu \frac{\partial F(w, t)}{\partial x}\right) .
$$

Let us introduce a new value $\varphi_{F}-$ «relative elasticity of moisture potential»:

$$
\varphi_{F}(w, t)=\varphi(w)+\frac{1}{\mu} \int_{0}^{w} \beta(\sigma) d \sigma / E_{t}(t) .
$$

Taking into consideration (20), moisture transfer differential equation (19) can be written as:

$$
\gamma_{0} \cdot\left(E_{t}(t) \frac{\partial \varphi_{F}(w, t)}{\partial w}\right)^{-1} \frac{\partial F(w, t)}{\partial \tau}=\frac{\partial}{\partial x}\left(\mu \frac{\partial F(w, t)}{\partial x}\right) .
$$

"Relative elasticity of moisture potential" $\varphi_{F}$ is a function of two variables : humidity and temperature. From a physics perspective $\varphi_{F}$ shows what fold total flow pressure value consisting of water vapor pressure and equivalent liquid moisture pressure, increase saturated water vapor pressure.

Graphic presentation of « relative elasticity of moisture potential» is proposed. For example, $\varphi_{F}$ for silica brick can be presented as (Fig. 2).

Let us introduce a new value $\xi_{F}-\langle$ relative potential capacity coefficient»:

$$
\xi_{F}(w, t)=\frac{d w}{d \varphi_{F}(w, t)} .
$$




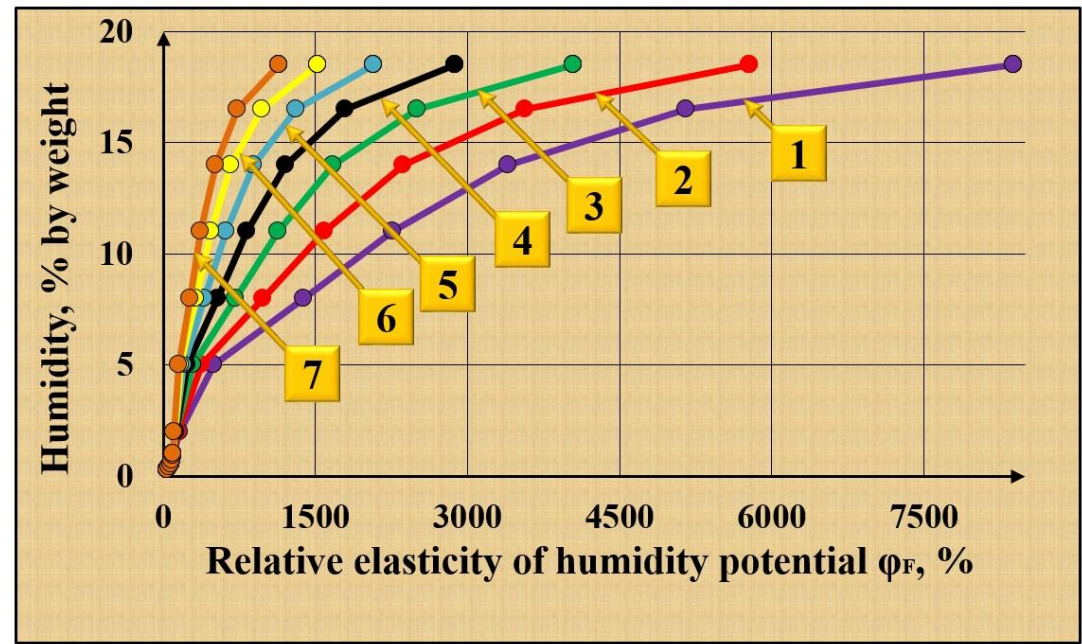

Fig. 2. Lime brick material humidity dependence on relative elasticity of moisture potential $\left(1--10^{\circ} \mathrm{C}\right.$ temperature; $2-5^{\circ} \mathrm{C}$ temperature; $3-0^{\circ} \mathrm{C}$ temperature; $4-5^{\circ} \mathrm{C}$ temperature; $5-10^{\circ} \mathrm{C}$ temperature; 6 $-15^{\circ} \mathrm{C}$ temperature; $7-20^{\circ} \mathrm{C}$ temperature).

To reduce moisture transfer differential equation to form of equation with constant coefficients, differential coefficients in (22) are replaced by finite differences:

$$
\xi_{F 0}(w, t)=\frac{\Delta w}{\Delta \varphi_{F}} .
$$

Taking (23) into consideration, moisture transfer differential equation can be written as:

$$
\gamma_{0} \cdot \frac{\xi_{F 0}(w, t)}{E_{t}(t)} \cdot \frac{\partial F(w, t)}{\partial \tau}=\frac{\partial}{\partial x}\left(\mu \frac{\partial F(w, t)}{\partial x}\right) .
$$

Let us introduce a new value $\kappa$ - «heat-to-humidity material property coefficient»:

$$
\kappa(w, t)=\frac{\mu}{\gamma_{0} \cdot \xi_{F 0}(w, t)} .
$$

Taking (25) into consideration, moisture transfer modified differential equation can be finally written as:

$$
\frac{\partial F(w, t)}{\partial \tau}=E_{t}(t) \frac{\partial}{\partial x}\left(\kappa(w, t) \frac{\partial F(w, t)}{\partial x}\right) .
$$

For equation (26) moisture exchange boundary conditions (15), (16) are set at enclosing structure boundaries, condition of moisture potential flow continuity (17) is set between layers. Calculation time is divided into timeframes one month long. Steady-state temperature distribution in the enclosing structure thickness is studied within each month (7). Moisture continuity is set between timeframes (8).

Temperature and moisture potential inside the enclosing structure are taken to be constant during all calculation period. Outside the enclosing structure moisture potential varies linearly according to climatological data during every timeframe: 


$$
F_{e x t}=m \cdot \tau+n .
$$

where $\mathrm{m}$ - boundary conditions slope ratio within a month; $n$ - boundary conditions graph rise within a month, $\mathrm{Pa}$.

\subsection{Development of moisture transfer problem solution for single-layer enclosing structures using discrete-continual approach}

For a single-layer enclosing structure moisture transfer differential equation (26) can be written as:

$$
\frac{\partial F(w, t)}{\partial \tau}=\kappa(w, t) \cdot E_{t}(t) \frac{\partial^{2} F(w, t)}{\partial x^{2}} .
$$

Let us use discrete-continual approach [29, 30] to the equation (28) (Fig. 3).

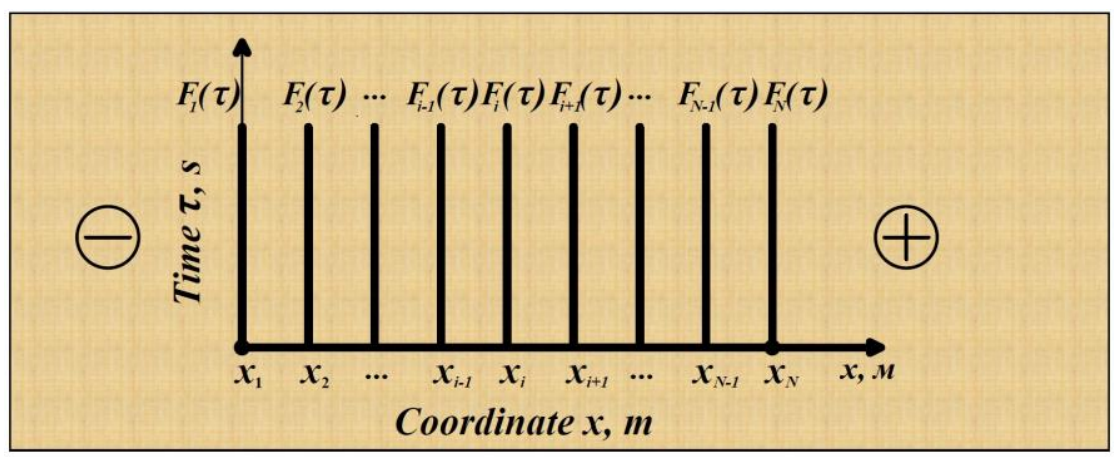

Fig. 3. Discrete-continual approximation of spatial-time domain of a single-layer enclosing structure.

Partial differential coefficients in X-direction are replaced by finite differences, and in time direction $\tau$ we are seeking a solution in form of a function. Taking boundary conditions (15), (16) into consideration we have simultaneous equations:

$$
\left\{\begin{array}{l}
\frac{\partial F_{1}}{\partial \tau}=\frac{\kappa}{h^{2}} \cdot E_{t 1} \cdot\left(-\left(1+\frac{\beta_{e x t}}{\mu} \cdot h\right) \cdot F_{1}+F_{2}\right)+\frac{\kappa}{h^{2}} \cdot E_{t 1} \cdot \frac{\beta_{e x t}}{\mu} \cdot h \cdot F_{e x t} \\
\frac{\partial F_{i}}{\partial \tau}=\frac{\kappa}{h^{2}} \cdot E_{t i} \cdot\left(F_{i-1}-2 \cdot F_{i}+F_{i+1}\right), i=2,3,4, \ldots, N-1 \\
\frac{\partial F_{N}}{\partial \tau}=\frac{\kappa}{h^{2}} \cdot E_{t N} \cdot\left(F_{N-1}-\left(1+\frac{\beta_{i n}}{\mu} \cdot h\right) F_{N}\right)+\frac{\kappa}{h^{2}} \cdot E_{t N} \cdot \frac{\beta_{i n}}{\mu} \cdot h \cdot F_{i n} \\
F(x, 0)=\grave{u}(x), 0 \leq x \leq l
\end{array} .\right.
$$

Simultaneous equations (29) represent a Cauchy problem in matrix form:

$$
\left\{\begin{array}{l}
\bar{F}_{\tau}^{\prime}=E_{t} \cdot A \cdot \bar{F}+p \cdot \tau \cdot \bar{L}+\bar{B} \\
\bar{F}(0)=\bar{\chi}, 0 \leq x \leq l
\end{array} .\right.
$$

where

$$
p=\kappa \cdot E_{t 1} \cdot \beta_{\text {ext }} \cdot h \cdot m /\left(h^{2} \cdot \mu\right)
$$




$$
\begin{gathered}
A=\frac{\kappa}{h^{2}} \cdot\left(\begin{array}{ccccc}
-\left(1+\beta_{\text {ext }} \cdot h / \mu\right) & 1 & 0 & 0 & 0 \\
1 & -2 & 1 & 0 & 0 \\
0 & \ddots & \ddots & \ddots & 0 \\
0 & 0 & 1 & -2 & 1 \\
0 & 0 & 0 & 1 & -\left(1+\beta_{i n} \cdot h / \mu\right)
\end{array}\right) \cdot E_{t}=\left(\begin{array}{ccccc}
E_{t 1} & 0 & 0 & 0 & 0 \\
0 & E_{t 2} & 0 & 0 & 0 \\
\ldots & \ldots & \ldots & \ldots & \ldots \\
0 & 0 & 0 & E_{t(N-1)} & 0 \\
0 & 0 & 0 & E_{t N}
\end{array}\right) \\
\bar{F}_{\tau}^{\prime}=\left(\begin{array}{c}
F_{1}^{\prime}(\tau) \\
F_{2}^{\prime}(\tau) \\
\cdots \\
F_{N-1}^{\prime}(\tau) \\
F_{N}^{\prime}(\tau)
\end{array}\right) \cdot \bar{F}=\left(\begin{array}{c}
1 \\
F_{1} \\
F_{2} \\
\cdots \\
F_{N-1} \\
F_{N}
\end{array}\right) \cdot \bar{L}=\left(\begin{array}{c}
\kappa \cdot E_{t 1} \cdot \beta_{e x t} \cdot h \cdot n /\left(h^{2} \cdot \mu\right) \\
0 \\
\ldots \\
0 \\
0
\end{array}\right) \cdot \bar{B}=\left(\begin{array}{c}
\ldots \\
0 \\
\kappa \cdot E_{t N} \cdot \beta_{i n} \cdot h \cdot F_{i n} /\left(h^{2} \cdot \mu\right)
\end{array}\right)
\end{gathered}
$$

Problem solution (17) can be written as:

$$
\begin{gathered}
\bar{F}=p \cdot\left(\left(E_{t} \cdot A\right)^{-2} \cdot e^{E_{t} \cdot A \cdot \tau}-\tau \cdot\left(E_{t} \cdot A\right)^{-1}-\left(E_{t} \cdot A\right)^{-2}\right) \cdot \bar{L}+\left(E_{t} \cdot A\right)^{-1}\left(e^{E_{t} \cdot A \cdot \tau}-E\right) \cdot \bar{B}+ \\
+e^{E_{t} \cdot A \cdot \tau} \cdot \bar{F}_{0} .
\end{gathered}
$$

where $E$ - unit matrix.

The proposed formula (32) allows to determine moisture potential distribution in any section of a single-layer enclosing structure at any moment of time under continuous control for temperature distribution.

\subsection{Development of moisture transfer problem solution for multi-layer enclosing structures using discrete-continual approach}

Let us consider discrete-continual approach application by the example of a double-layer enclosing structure.

Similarly, let us use discrete-continual approach on equation (26) for a multi-layer enclosing structure (fig. 4).

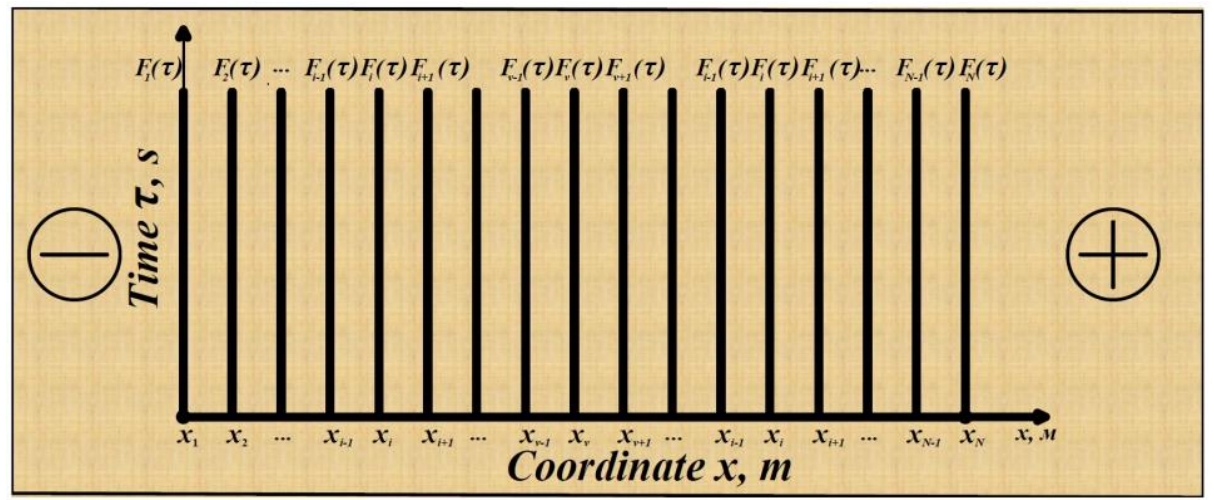

Fig. 4. Discrete-continual approximation of spatial-time domain of a multi-layer enclosing structure.

Taking into account boundary conditions (15), (16) and moisture potential flow equality between layers (17) we have the following simultaneous equations: 


$$
\left\{\begin{array}{c}
\frac{\partial F_{1}}{\partial \tau}=\frac{\kappa_{1}}{h_{1}^{2}} \cdot E_{t 1} \cdot\left(-\left(1+\frac{\beta_{e x t}}{\mu_{1}} \cdot h_{1}\right) \cdot F_{1}+F_{2}\right)+\frac{\kappa_{1}}{h_{1}^{2}} \cdot E_{t 1} \cdot \frac{\beta_{e x t}}{\mu_{1}} \cdot h_{1} \cdot F_{e x t}, i=1 \\
\frac{\partial F_{i}}{\partial \tau}=\frac{\kappa_{1}}{h_{1}^{2}} \cdot E_{t i} \cdot\left(F_{i-1}-2 \cdot F_{i}+F_{i+1}\right), i=2,3,4, \ldots, v-1 \\
\frac{\partial F_{v}}{\partial \tau}=d_{v-1} \cdot F_{v-1}-d_{v} \cdot F_{v}+d_{v+1} \cdot F_{v+1}, i=v \\
\frac{\partial F_{i}}{\partial \tau}=\frac{\kappa_{2}}{h_{2}^{2}} \cdot E_{t i} \cdot\left(F_{i-1}-2 \cdot F_{i}+F_{i+1}\right), i=v+1, v+2, \ldots, N-1 \\
\frac{\partial F_{N}}{\partial \tau}=\frac{\kappa_{2}}{h_{2}^{2}} \cdot E_{t N} \cdot\left(F_{N-1}-\left(1+\frac{\beta_{i n}}{\mu_{2}} \cdot h_{2}\right) F_{N}\right)+\frac{\kappa_{2}}{h_{2}^{2}} \cdot E_{t N} \cdot \frac{\beta_{i n}}{\mu_{2}} \cdot h_{2} \cdot F_{i n}, i=N \\
F(x, 0)=\grave{u}(x), 0 \leq x \leq l \\
\quad d_{v-1}=0,024 \cdot 2 \cdot E_{t v} \cdot \mu_{1} /\left(h_{1}\left(h_{1} \cdot \gamma_{01} \cdot \xi_{F 01}+h_{2} \cdot \gamma_{02} \cdot \xi_{F 02}\right)\right) . \\
\quad d_{v}=0,024 \cdot 2 \cdot\left(\mu_{1} / h_{1}+\mu_{2} / h_{2}\right) /\left(h_{1} \cdot \gamma_{01} \cdot \xi_{F 01}+h_{2} \cdot \gamma_{02} \cdot \xi_{F 02}\right) . \\
\quad d_{v+1}=0,024 \cdot 2 \cdot E_{t v} \cdot \mu_{2} /\left(h_{2}\left(h_{1} \cdot \gamma_{01} \cdot \xi_{F 01}+h_{2} \cdot \gamma_{02} \cdot \xi_{F 02}\right)\right) .
\end{array}\right.
$$

where $i$ - enclosing structure section number; $h_{1}$ - first layer partition step (closer to outer surface) of the enclosing structure in coordinate $\mathrm{x}, \mathrm{m} ; h_{2}-$ second layer partition step (closer to inner surface) of the enclosing structure in coordinate $\mathrm{x}, \mathrm{m} ; \gamma_{01}-$ dry material density in the first layer of an enclosing structure, $\mathrm{kg} / \mathrm{m}^{3} ; \gamma_{02}$ - dry material density in the second layer of an enclosing structure, $\mathrm{kg} / \mathrm{m}^{3} ; \xi_{F 01}$ - relative potential capacity of the first layer material of an enclosing structure, $\mathrm{kg} / \mathrm{kg} ; \xi_{F 02}$ - relative potential capacity of the second layer material of an enclosing structure, $\mathrm{kg} / \mathrm{kg} ; \kappa_{1}-$ «heat-to-humidity material property coefficient» of the first layer of an enclosing structure, $m^{2} /(s \cdot P a)$; $\kappa_{2}-$ «heat-to-humidity material property coefficient» of the second layer of an enclosing structure, $m^{2} /(s \cdot P a) ; \grave{u}$ - initial moisture potential distribution, $\mathrm{Pa}$. form:

Simultaneous equations (33), (34), (35), (36) represent a Cauchy problem in matrix

$$
\left\{\begin{array}{l}
\bar{F}_{\tau}^{\prime}=\left(G+K \cdot E_{t} \cdot A\right) \cdot \bar{F}+p \cdot \tau \cdot \bar{L}+\bar{B} \\
\bar{F}_{0}=\bar{\chi}, 0 \leq x \leq l
\end{array} .\right.
$$

where

$$
p=\kappa_{1} \cdot E_{t 1} \cdot \beta_{e x t} \cdot h_{1} \cdot m /\left(h_{1}^{2} \cdot \mu_{1}\right)
$$




$$
\begin{aligned}
& \bar{F}_{\tau}^{\prime}=\left(\begin{array}{c}
F_{1}^{\prime}(\tau) \\
F_{2}^{\prime}(\tau) \\
\ldots \\
F_{N-1}^{\prime}(\tau) \\
F_{N}^{\prime}(\tau)
\end{array}\right) \cdot G=\left(\begin{array}{ccccc}
0 & 0 & 0 & 0 & 0 \\
0 & 0 & 0 & 0 & 0 \\
0 & d_{v-1} & d_{v} & d_{v+1} & 0 \\
0 & \ldots & \ldots & \ldots & 0 \\
0 & 0 & 0 & 0 & 0
\end{array}\right) \cdot K=\left(\begin{array}{ccccc}
\kappa_{1} / h_{1}^{2} & 0 & 0 & 0 & 0 \\
0 & \kappa_{1} / h_{1}^{2} & 0 & 0 & 0 \\
0 & \ldots & 0 & \ldots & 0 \\
0 & 0 & 0 & \kappa_{2} / h_{2}^{2} & 0 \\
0 & 0 & 0 & 0 & \kappa_{2} / h_{2}^{2}
\end{array}\right) \cdot \bar{\chi}=\left(\begin{array}{c}
F_{e x t} \\
F_{e x t} \\
\ldots \\
F_{e x t} \\
F_{e x t}
\end{array}\right) . \\
& E_{t}=\left(\begin{array}{ccccc}
E_{t 1} & 0 & 0 & 0 & 0 \\
0 & E_{t 2} & 0 & 0 & 0 \\
\cdots & \cdots & \cdots & \cdots & \cdots \\
0 & 0 & 0 & E_{t(N-1)} & 0 \\
0 & 0 & 0 & 0 & E_{t N}
\end{array}\right) \cdot A=\left(\begin{array}{ccccc}
-\left(1+\beta_{e x t} \cdot h_{1} / \mu_{1}\right) & 1 & 0 & 0 & 0 \\
1 & -2 & 1 & 0 & 0 \\
0 & \ddots & \ddots & \ddots & 0 \\
0 & 0 & 1 & -2 & 1 \\
0 & 0 & 0 & 1 & -\left(1+\beta_{\text {in }} \cdot h_{2} / \mu_{2}\right)
\end{array}\right) \text {. } \\
& \bar{F}=\left(\begin{array}{c}
F_{1} \\
F_{2} \\
\cdots \\
F_{N-1} \\
F_{N}
\end{array}\right) \cdot \bar{L}=\left(\begin{array}{c}
1 \\
0 \\
\ldots \\
0 \\
0
\end{array}\right) \cdot \bar{B}=\left(\begin{array}{c}
\kappa_{1} \cdot E_{t 1} \cdot \beta_{e x t} \cdot h_{1} \cdot n /\left(h_{1}^{2} \cdot \mu_{1}\right) \\
0 \\
\ldots \\
0 \\
\kappa_{2} \cdot E_{t N} \cdot \beta_{i n} \cdot h_{2} \cdot F_{i n} /\left(h_{2}^{2} \cdot \mu_{2}\right)
\end{array}\right) . \bar{F}_{0}=\left(\begin{array}{c}
F_{1}(0) \\
F_{2}(0) \\
\ldots \\
F_{N-1}(0) \\
F_{N}(0)
\end{array}\right) .
\end{aligned}
$$

The equation (37) is a Cauchy problem, which can be solved in matrix form:

$$
\begin{aligned}
& \bar{F}=p \cdot\left(\left(G+K \cdot E_{t} \cdot A\right)^{-2} \cdot e^{\left(G+K \cdot E_{t} \cdot A\right) \cdot \tau}-\tau \cdot\left(G+K \cdot E_{t} \cdot A\right)^{-1}-\left(G+K \cdot E_{t} \cdot A\right)^{-2}\right) \cdot \bar{L}+ \\
& +\left(G+K \cdot E_{t} \cdot A\right)^{-1}\left(e^{\left(G+K \cdot E_{t} \cdot A\right) \cdot \tau}-E\right) \cdot \bar{B}+e^{\left(G+K \cdot E_{t} \cdot A\right) \cdot \tau} \cdot \bar{F}_{0}
\end{aligned}
$$

The proposed formula (32) allows to determine moisture potential distribution in any section of a multi-layer enclosing structure at any moment of time under continuous control for temperature distribution.

\section{Results}

\subsection{Moisture regime study of a single-layer enclosing structure by means of various methods of moisture potential theory}

To solve the problem, moisture regime of a single-layer enclosing structure has been studied by means of various methods of moisture potential theory: engineering quasistationary method, unsteady-state method, and the proposed discrete-continual method. Single-layer enclosing structure made of $0.6 \mathrm{~m}$ aerated concrete $\left(400 \mathrm{~kg} / \mathrm{m}^{3}\right.$ density) in Moscow climatic conditions has been used for calculation. Air temperature $20^{\circ} \mathrm{C}$ and relative air humidity $50 \%$ have been maintained in the room.

Moisture potential distribution in engineering quasi-stationary method has been determined analytically according to equation (18). Moisture potential distribution in unsteady-state method has been determined numerically by finite difference method using an explicit difference scheme. Moisture potential distribution in discrete-continual method has been determined according to the proposed formula (32). 
Moisture regime of a single-layer enclosing structure is presented in the diagram (fig. 5).

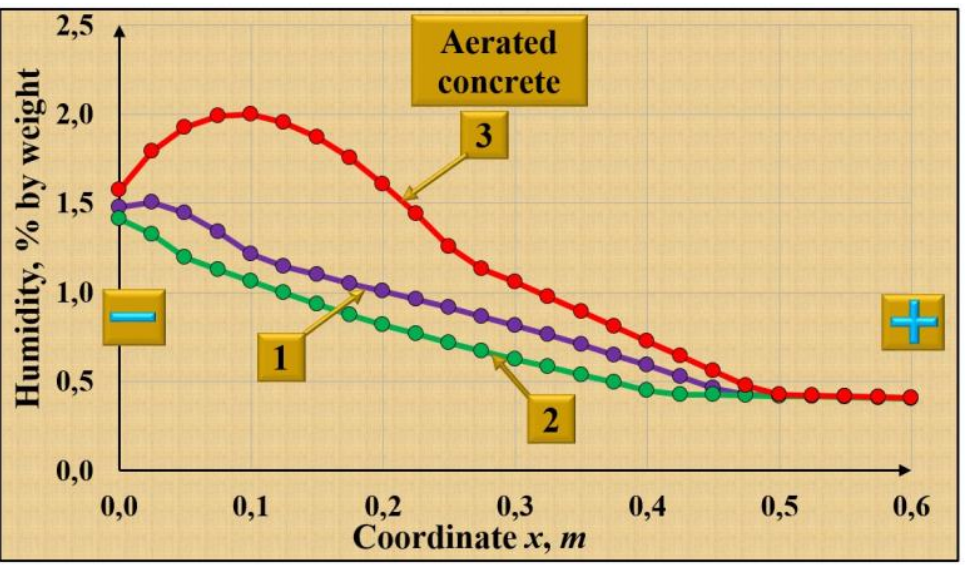

Fig. 5. Comparison of moisture potential theory methods for moisture regime calculation of singlelayer enclosing structure in January $(1$ - moisture distribution in enclosing structure according to unsteady-state method, 2 - moisture distribution in enclosing structure according to discretecontinuous method, 3 - moisture distribution in enclosing structure according to engineering method).

\subsection{Moisture regime study of multi-layer enclosing structures by means of various methods of moisture potential theory}

Similarly, moisture regime of a multi-layer enclosing structure has been studied. Doublelayer enclosing structure made of 380 -mm clay brick basement and 120 -mm lime brick cladding located in Moscow climatic conditions has been used for calculation. Air temperature $22{ }^{\circ} \mathrm{C}$ and relative air humidity $60 \%$ have been maintained in the room. $6)$.

Moisture regime of a double-layer enclosing structure is presented in the diagram (fig.

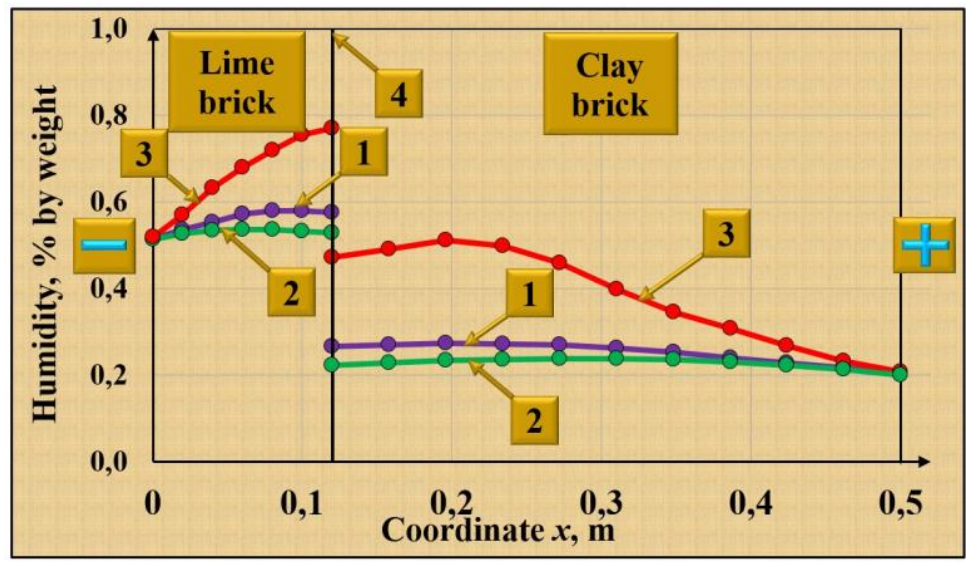

Fig. 6. Comparison of moisture potential theory methods for moisture regime calculation of multilayer enclosing structure in January $(1$ - moisture distribution in enclosing structure according to unsteady-state method, 2 - moisture distribution in enclosing structure according to discretecontinuous method, 3 - moisture distribution in enclosing structure according to engineering method, 4 - border between layers). 
The diagram (Fig. 6) shows moisture distribution of a double-layer enclosing structure. Formula (39) can be also applied for moisture regime assessment of multi-layer enclosing structures having more than two layers.

\section{Discussion}

Analysis of obtained results demonstrates that the greatest moisture value both for singlelayer (fig. 5) and multi-layer (fig. 6) enclosing structure is achieved by quasi-stationary calculation method. The reason is: conditions on enclosing structure boundaries do not vary for a long time, moisture potential distribution becomes steady-state, and moistening achieves a certain maximum value determined by boundary conditions.

Moisture distribution obtained by unsteady-state calculation method takes into account wetting process response time. Presented results show that the structure has no time to moisten enough up to steady-state moisture potential distribution.

The proposed discrete-continual calculation method allows to obtain quantitative and qualitative moisture distribution results similar to results obtained by unsteady-state calculation method. The benefit of this method is the results obtained analytically, which makes calculation considerably easier.

\section{Conclusions}

New efficient method using discrete-continual approach has been developed for unsteadystate moisture regime study of single-layer and multi-layer enclosing structures. Formulas of moisture potential inside the enclosing structure have been derived. Moisture regime calculation by means of the proposed discrete-continual method gives results close to the well-known unsteady-state method. This, on the one hand, confirms accuracy of the developed method, and on the other, enables to use this method in engineering practice.

\section{References}

1. Z. Wu, H.S. Wong, N.R. Buenfeld, Cement and concrete research 98, 136-154 (2017)

2. E. Zvicevicius, A. Raila, A. Cipliene, Z. Cerniauskiene, Z. Kadziuliene, V. Tilvikiene 119, 185-192 (2018)

3. F. Georget, J.H. Prevost, B. Huet, Cement and concrete research 104, 1-12 (2018)

4. Z.C. Liu, W. Hansen, F.Z. Wang, Construction and building materials 158, 181-188 (2018)

5. X.X. Li, S.H. Chen, Q. Xu, Y. Xu, Journal of materials in civil engineering 30, 1-15 (2018)

6. Z. Pavlik, M. Pavlikova, L. Fiala, Z. Suchorab, H. Sobczuk, R. Cerny, Thermophysics 2010, 243-247 (2010)

7. Z. Suchorab, H. Sobczuk, M. Skwarczynski, Thermophysics 2010, 283-289 (2010)

8. Z. Suchorab, D. Barnat-Hunek, H. Sobczuk, Journal of building engineering 18, 111120 (2011)

9. D. Belkharchouche, A. Chaker, International journal of hydrogen energy 41, 71197125 (2016)

10. A. Hoseini, A. Bahrami, Journal of building engineering 13, 107-115 (2017) 
11. H.Q. Jin, X.L. Yao, L.W. Fan, X. Xu, International journal of heat and mass transfer 92, 589-602 (2017)

12. F. Girault, F. Perrier, Science of the total environment 433, 247-263 (2012)

13. A.S. Petrov, V.N. Kupriyanov, International journal of pharmacy and technology 8 , 11248-11256 (2016)

14. P. Perre, F. Pierre, J. Casalinho, M. Ayouz, Drying technology 33, 1068-1075 (2015)

15. N. Potzsch, N. Ruther, Bauphysik 31, 106-109 (2009)

16. M.I. Nizovtsev, A.N. Sterlyagov, V.I. Terekhov, Concrete materials: properties, performance and applications, 397-429 (2009)

17. G.H. Galbraith, D.J. Kelly, R.C. McLean, Research in building physics, 249-254 (2003)

18. N. Shukla, D. Kumar, D. Elliott, J. Kosny, Next-generation thermal insulation challenges and opportunities 1574, 66-80 (2014)

19. S. Rubene, M. Vilnitis, J. Noviks, Impact of External Heat Concrete Masonry Constructions 2nd international conference on innovative materials, structures and technologies 96, 1-8 (2015)

20. Z. Suchorab, H. Sobczuk, G. Lagod, Thermophysics 2016: 21st international meeting 2016 1752, 1-7 (2016)

21. O. Sass, H.A. Viles, Limestone in the built environment: present day challenges for the preservation of the past 331, 237-249 (2010)

22. J.A. Eklund, H. Zhang, H.A. Viles, T. Curteis, International journal of architectural heritage 7, 207-224 (2013)

23. B. Vavrovic, Advanced Materials Research 855, 97-101 (2014)

24. L. Skerget, A. Tadeu, J. Ravnik, Engineering analysis with boundary elements 74, 2433 (2017)

25. S. Lal, F. Lucci, T. Defraeye, L.D. Poulikakos, M.N. Partl, D. Derome, J. Carmeliet, International journal of thermal sciences 123, 86-98 (2018)

26. Y.C. Tang; J.C. Min, X.M. Wu, International journal of heat and mass transfer 116, 371-376 (2018)

27. J. Arfvidsson, J. Claesson, Building and environment 35, 519-536 (2000)

28. V.G. Gagarin, V.K. Akhmetov, K.P. Zubarev, MATEC Web of Conferences 170, 1-6 (2018)

29. V.N. Sidorov, S.M. Matskevich, Procedia Engineering 111, 726-733 (2015)

30. V.N. Sidorov, S.M. Matskevich, Key Engineering Materials 685, 211-216 (2016) 